\title{
Stable, efficient p-type doping of graphene by nitric
}

\section{acid}

Lorenzo D'Arsié,,$^{\dagger *}$ Santiago Esconjauregui, ${ }^{\dagger}$ Robert S. Weatherup ${ }^{\dagger}$ Xingyi Wu,,$^{\dagger}$ William Arter ${ }^{\dagger}$ Hisashi Sugime, ${ }^{\dagger}$ Cinzia Cepek, ${ }^{+}$and John Robertson ${ }^{\dagger}$

${ }^{\dagger}$ Department of Engineering, University of Cambridge, Cambridge CB3 0FA, United Kingdom ${ }^{\dagger}$ Istituto Officina dei Materiali-CNR, Laboratorio TASC, Trieste I-34149, Italy

KEYWORDS: Graphene, $\mathrm{HNO}_{3}$, doping, stability

\begin{abstract}
We systematically dope monolayer graphene with different concentrations of nitric acid over a range of temperatures, and analyze the variation of sheet resistance under vacuum annealing up to $300{ }^{\circ} \mathrm{C}$. The optimized $\mathrm{HNO}_{3}$ doping conditions yield sheet resistances as low as $180 \Omega / \mathrm{sq}$, which, under vacuum annealing, is significantly more stable than previously reported values. Raman and photoemission spectroscopy show that this stable graphene doping occurs by a bimodal mechanism. At mild conditions the dopants are weakly bonded to graphene, but at high acid temperatures and concentrations, the doping is higher and more stable upon post-doping annealing, without causing significant lattice damage. This work shows that large, stable hole concentrations can be induced by transfer doping in graphene.
\end{abstract}




\section{Introduction}

Graphene exhibits remarkable electrical, optical and mechanical properties, ${ }^{1,2}$ which may be advantageous for various applications, ${ }^{3}$ including electronics, ${ }^{4}$ photovoltaics, ${ }^{5}$ energy storage ${ }^{6}$ lighting $^{7}$ and displays. ${ }^{8}$ In particular, graphene is envisaged as an environmental-friendly and flexible substitute for indium tin oxide (ITO) as transparent conductor, ${ }^{9,10}$ but obtaining low and stable sheet resistances remains difficult. Pristine graphene exhibits high carrier mobility, but its intrinsic carrier density is close to zero, making its conductivity uncompetitive with ITO. Doping is therefore necessary and can be achieved by various means, such as substitution of atoms in the lattice, ${ }^{11,12}$ functionalization, ${ }^{13}$ and adsorption of atoms and molecules. ${ }^{14,15}$ Of these, substitutional doping in 2D crystals significantly alters the graphene lattice perturbing the unique properties of graphene, ${ }^{1,12}$ hence less invasive techniques, like adsorptive doping, are more desirable. The ideal doping induces a high charge carrier density $\left(>10^{13} \mathrm{~cm}^{-2}\right)$, remains stable in time across a broad temperature range, and is transparent and homogeneous. Thermal stability is particularly important in optoelectronic devices where significant power may be dissipated. However, to date efficient charge carrier transfer and stability have not been simultaneously achieved. Stable doping can be obtained, for instance, by evaporating high work function oxides like $\mathrm{MoO}_{3}{ }^{16,14,17,7}$ which, whilst effective for carbon nanotube forests where the resistance is lowered by two orders of magnitude, ${ }^{18}$ only show a two-fold reduction in sheet resistance (from $\sim 1210$ to $\sim 600 \Omega / \mathrm{sq}$ ) for monolayer graphene. ${ }^{14}$ The charge transfer is thus simply not sufficient for many of the envisioned applications of graphene as a transparent conductor.

In 2010, Bae et al. ${ }^{8}$ reported that graphene doped with $\mathrm{HNO}_{3}$ exhibits a remarkably low sheet resistance of $\sim 125 \Omega /$ sq for monolayer graphene. Subsequent studies however showed unstable behavior in air at room temperature (RT) over days ${ }^{15}$ or on vacuum annealing. ${ }^{7}$ The poor stability 
of the $\mathrm{HNO}_{3}$ doping was attributed to the volatility of adsorbed molecules, but a full understanding of the surface chemistry after doping is still lacking. Until now, the stability of $\mathrm{HNO}_{3}$-doped graphene was measured on samples with various layer numbers, and employing diverse doping procedures and techniques for stability evaluation, ${ }^{7,15}$ making comparisons of these results difficult. In this report, we systematically analyze the stability of $\mathrm{HNO}_{3}$-doped graphene, and demonstrate a doping procedure that lowers the sheet resistance of monolayer graphene to $180 \Omega /$ sq and shows stable behavior following annealing in vacuum up to $130{ }^{\circ} \mathrm{C}$. These results represent a step towards graphene with a sheet resistance, which is both stable and low enough to be competitive with ITO. Furthermore, our results provide more general insights into the chemical effects of $\mathrm{HNO}_{3}$ treatments on graphitic materials, of relevance to processes such as the removal of impurities from carbon nanotubes, ${ }^{19}$ and the preparation of graphene oxide. ${ }^{20}$ We also contrast the behavior of $\mathrm{HNO}_{3}$ to react with defects in carbon systems, as for example in the purification of carbon nanotubes, ${ }^{21}$ with the pure transfer doping which does not form new defects or react with existing ones.

\section{Experimental section}

As a substrate for device fabrication, we use silicon wafers with $300 \mathrm{~nm}$ of $\mathrm{SiO}_{2}$, on which we pattern electrodes $(\mathrm{Cr} / \mathrm{Au} 5 / 45 \mathrm{~nm})$ by electron beam lithography and UVIII resist. Monolayer graphene is grown by chemical vapor deposition on an Alfa Aesar Cu foil (25 $\mu \mathrm{m}, 99.8 \%$ purity) pre-treated by electropolishing in a home-built cell. The foil is heated up at temperature in Argon

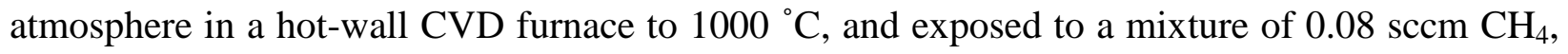
$10 \mathrm{sccm} \mathrm{H}_{2}$, and $490 \mathrm{sccm}$ Ar for $140 \mathrm{~min}$ to grow monolayer graphene on the surface. The foil is then cooled down in a mixture of $10 \mathrm{sccm} \mathrm{H}_{2}$ and $490 \mathrm{sccm}$ Ar. More detail can be found in a 
recent publication. ${ }^{22}$ The graphene is then transferred to patterned electrodes by a wet transfer process, using a poly-methyl methacrylate (PMMA) support layer and an aqueous solution of ammonium persulfate $(0.1 \mathrm{M})$ as etchant. The graphene is then patterned using electron beam lithography with UVIII resist followed by etching with oxygen plasma. To perform resistivity measurements, we fabricate 4 point probe devices with $4 \mu \mathrm{m}$ wide graphene channels and electrode distances of 5/10/5 $\mu \mathrm{m}$. For mobility measurements, we fabricate Hall devices with Van der Pauw geometry on a $50 \times 50 \mu \mathrm{m}^{2}$ piece of graphene with $2.5 \times 2.5 \mu \mathrm{m}^{2}$ contacts at the corners. To remove residues attached to graphene during transfer and device fabrication, we perform vacuum annealing at $180{ }^{\circ} \mathrm{C}$ and $10^{-7}$ mbar for 1 hour. ${ }^{23}$

Acid treatment is performed by dipping the chip into $65 \% \mathrm{HNO}_{3}$ diluted in DI water to achieve concentrations from $15-65 \%$. To control the acid temperature, $\sim 5 \mathrm{ml}$ of acid in a $10 \mathrm{ml}$ beaker is placed on a hotplate, while the temperature of the liquid is checked by a type $\mathrm{K}$ thermocouple. The sample is then blown with dry nitrogen. Annealing of the sample after doping is performed at $10^{-7}$ mbar with the temperature measured with a type $\mathrm{K}$ thermocouple placed on the heater for the samples used for electrical and Raman measurements, while at $10^{-8}$ mbar with the type $\mathrm{K}$ thermocouple placed on the sample for the X-ray photoemission spectroscopy (XPS) measurements.

XPS measurements are performed on a Mg X-ray source $(\mathrm{h} v=1253.6 \mathrm{eV})$. The photoelectrons are detected with an energy resolution of $\sim 0.8 \mathrm{eV}$ in normal emission geometry. The spectra are fitted with Doniach-Šunjić profiles convoluted with Gaussians after subtracting a Shirley background. The $\mathrm{N}: \mathrm{C}$ ratio is calculated by evaluating the areas of the $\mathrm{N} 1 s$ and $\mathrm{C}$ 1s photoemission spectra, normalized by the photoionization cross section area of the different elements. The $\mathrm{N}$ atom density is then extracted by the known atomic density of $\mathrm{C}$ atoms in 
graphene (calculated using $0.142 \mathrm{~nm}$ as $\mathrm{C}-\mathrm{C}$ bond). If not stated otherwise, Raman spectra are acquired by a $532 \mathrm{~nm}$ laser at $\sim 1.5 \mathrm{~mW}$ with a $50 \times$ objective (spot size of $\sim 1 \mu \mathrm{m}$ ). The Raman peaks are fitted with Lorentzian profiles.

\section{Results}

Figure 1 shows that higher $\mathrm{HNO}_{3}$ concentrations and temperatures are more effective in lowering the sheet resistance of graphene and achieving a more stable resistance. The plots show the change in resistance obtained by dipping the sample in $\mathrm{HNO}_{3}$ for different (a) acid concentrations, (b) exposure times, and (c) acid temperatures. The change in resistance with acid concentration is measured for samples exposed to acid for 5 min at RT. The measurements show a similar decrease in resistance for both $15 \%$ and $30 \% \mathrm{HNO}_{3}$ concentrations to $\sim 0.2 \times$ that of the pristine graphene, while at $65 \%$ concentration the resistance halves again to give a sheet resistance $0.1 \times$ that of the pristine graphene. The doping shows no significant dependence on exposure time for our timescales, as $15 \mathrm{~s}$ of dipping time in $65 \% \mathrm{HNO}_{3}$ already reaches a resistance value of $0.12 \times$ the original value, while at 1,5 , and $20 \mathrm{~min}$ the ratio saturates at 0.10 . A further decrease in resistance occurs by increasing the temperature of the acid solution. Keeping both concentration and dipping time constant at $65 \%$ and $5 \mathrm{~min}$, the resistance decreases to $0.07 \times$ the original resistance when the acid solution is at $52{ }^{\circ} \mathrm{C}$. These ratios were consistently observed for several devices with different initial sheet resistances in the range [2600-3500] $\Omega /$ sq. After pre-annealing in vacuum at $180{ }^{\circ} \mathrm{C}$ for $1 \mathrm{~h}$, the average sheet resistance is $3000 \Omega / \mathrm{sq}$, while after doping with $65 \%$ acid for $5 \mathrm{~min}$ the sheet resistance decreases to $\sim 310$ $\Omega /$ sq when the acid is at RT and to $\sim 210 \Omega / \mathrm{sq}$ when at $52{ }^{\circ} \mathrm{C}$. The lowest individual-device sheet resistance measured is $180 \Omega / \mathrm{sq}$, after a hot acid treatment. There is a contrast between the one 
order of magnitude reduction in graphene resistance by $\mathrm{HNO}_{3}$ treatment reported here and the more modest reductions reported elsewhere, e.g. by three times by Kasry et al. ${ }^{24}$ This can be explained by the higher resistive baseline of pre-annealed graphene, the number of graphene layers analyzed and the optimization of the doping procedure herein.

Figures $1(\mathrm{~d}, \mathrm{e}, \mathrm{f})$ show the fraction of sheet resistance regained upon heating in vacuum to $70{ }^{\circ} \mathrm{C}$, $130{ }^{\circ} \mathrm{C}, 200{ }^{\circ} \mathrm{C}$ and $300{ }^{\circ} \mathrm{C}$, i.e. the increase in resistance for post-annealing over the reduction in resistance upon doping. Diluted acid (15\% and 30\%) produces samples that are less stable than that doped with $65 \%$ acid solution. The difference is particularly evident after the $200{ }^{\circ} \mathrm{C}$ annealing which regains nearly $\sim 40 \%$ of the sheet resistance reduction achieved by low concentration acid dipping, while a recovery of just $13 \%$ occurs for more concentrated acid. The stability varies little with dipping time, but it depends strongly on the acid temperature. When graphene is doped with $65 \% \mathrm{HNO}_{3}$ for $5 \mathrm{~min}$ at $52{ }^{\circ} \mathrm{C}$, the doping is maintained even on vacuum annealing up to $130{ }^{\circ} \mathrm{C}$. The percentage of resistance recovery at $130{ }^{\circ} \mathrm{C}$ is less than $2 \%$ and corresponds to an absolute change in sheet resistance from $\sim 210$ to $\sim 250 \Omega / \mathrm{sq}$.

Figure 2 shows the trend of graphene mobility and charge carrier density after transfer, doping, and post-annealing at $200{ }^{\circ} \mathrm{C}$. The data is measured on 6 devices fabricated on three different chips, doped with low and high $\mathrm{HNO}_{3}$ concentration (22\% and 65\%) at RT, and high concentration $(65 \%)$ at $52{ }^{\circ} \mathrm{C}$. The as-transferred graphene (before pre-annealing) presents an average Hall mobility of $\sim 5500 \mathrm{~cm}^{2} \mathrm{~V}^{-1} \mathrm{~s}^{-1}$ and charge carrier density of $\sim 1.5 \times 10^{12} \mathrm{~cm}^{-2}$. The doping increases the charge carrier density considerably up to $\sim 4 \times 10^{13} \mathrm{~cm}^{-2}$ in the case of heated acid, but the mobility decreases to below $900 \mathrm{~cm}^{2} \mathrm{~V}^{-1} \mathrm{~s}^{-1}$. Finally, when post-annealed in vacuum, both mobility and charge carrier density partially recover, increasing and decreasing their values respectively. It is interesting to note that the mobility of the three samples does not diverge 
significantly for the different concentrations or temperatures of acid, while the charge carrier density behaves as the conductivity, i.e. it is higher and more stable for higher concentrations and temperatures of acid. We have not included the results on pre-annealed samples, since the measurements on different devices show large variations. The possible reason for this may relate to the Fermi energy being very close to the Dirac point. The residual surface contamination may therefore create regions of $p$-type and $n$-type doping (charge puddles), ${ }^{25}$ and thus the assumption of uniform doping in Van der Pauw Hall measurements no longer holds.

Figure $3 \mathrm{a}$ confirms the non-destructive nature of $\mathrm{HNO}_{3}$ doping. The Raman spectra of preannealed graphene show $\mathrm{I}_{2 \mathrm{D}} / \mathrm{I}_{\mathrm{G}}$ ratios of $\sim 2.09$, and $2 \mathrm{D}$ peak FWHM of $\sim 28 \mathrm{~cm}^{-1}$, which are typical values for monolayer graphene transferred on silicon dioxide ${ }^{26,27}$. After doping with low concentration $\mathrm{HNO}_{3}(23 \%)$, high concentration acid (65\%) and high concentration heated acid $\left(52{ }^{\circ} \mathrm{C}\right)$, the $\mathrm{I}_{2 \mathrm{D}} / \mathrm{I}_{\mathrm{G}}$ ratio decreases to $1.77,1.69$, and 1.59 respectively (still large), the $\mathrm{G}$ peak position shifts from $1590 \mathrm{~cm}^{-1}$ to $1592 \mathrm{~cm}^{-1}, 1593 \mathrm{~cm}^{-1}, 1594 \mathrm{~cm}^{-1}$ and the 2D peak wavenumber shifts from $2681 \mathrm{~cm}^{-1}$ to $2682 \mathrm{~cm}^{-1}, 2683 \mathrm{~cm}^{-1}, 2685 \mathrm{~cm}^{-1}$ respectively. These variations in the Raman spectra are consistent with increasing doping. ${ }^{28}$ All spectra have a small $\mathrm{D}$ peak $\left(\mathrm{I}_{\mathrm{D}} / \mathrm{I}_{\mathrm{G}}\right.$ ratio of $\sim 0.03$ ), which indicates the high crystalline quality of the graphene ${ }^{29}$ and the nondestructive nature of $\mathrm{HNO}_{3}$ doping.

Figure 4a shows the $\mathrm{C} 1 s$ spectrum of graphene before and after doping, fitted to resolve the contributing components. Before doping, the spectrum shows a main graphitic component and three smaller peaks at higher binding energies. The main peak is due to C-C $s p^{2}$ bonds in the graphene layer, while the other peaks can be attributed to $s p^{3}$ carbon, $\mathrm{C}-\mathrm{OH}$ and $\mathrm{C}=\mathrm{O}$, corresponding to both defects in the graphene layer and to polymer residue. ${ }^{30}$ After dipping the sample in $65 \% \mathrm{HNO}_{3}$ at $52{ }^{\circ} \mathrm{C}$ the higher binding energy components become $10 \%$ weaker, and 
all the peaks shift by $\sim 0.55 \mathrm{eV}$ to lower binding energies. The reduction of intensity of the nongraphitic peaks can be attributed to cleaning of polymer contamination from the graphene surface, as in Kasry et al., ${ }^{24}$ who observed higher transparency after $\mathrm{HNO}_{3}$ treatment. The shift indicates a band bending towards $p$-doping. The doping is also confirmed by the increase in asymmetry of the graphitic peak, attributable to an increase in charge carrier density. Figure $4 \mathrm{~b}$ shows the $\mathrm{N} 1 s$ spectra of graphene doped with $20 \% \mathrm{HNO}_{3}$ at $\mathrm{RT}$ and $65 \% \mathrm{HNO}_{3}$ at $\mathrm{RT}$ and 52 ${ }^{\circ} \mathrm{C}$, all of which have been post-annealed at $200{ }^{\circ} \mathrm{C}$. The spectra of doped graphene (dark green and dark blue) show two main components at $\sim 400 \mathrm{eV}$ and $\sim 406 \mathrm{eV}$, which can be attributed to nitrogen close to carbon atoms and $\mathrm{NO}_{\mathrm{x}}$ radicals, respectively. The peak at $\sim 400 \mathrm{eV}$ is already detected in the pre-annealed (undoped) sample, as in previous reports on other graphitic materials like graphite ${ }^{31}$ or single-walled carbon nanotubes (SWCNTs). ${ }^{32}$ The peak at $\sim 406 \mathrm{eV}$, seen in $\mathrm{HNO}_{3}$ treated graphite and SWCNTs, has to our knowledge not been previously reported for monolayer or few-layer graphene. ${ }^{33}$ Upon annealing the peak intensity at $\sim 406 \mathrm{eV}$ lowers, while the $\mathrm{C}-\mathrm{N}$ peak remains constant. The ratio between nitrogen and carbon atoms on the surface does not exceed $2 \%$, even for the case of $65 \%$ concentrated acid at RT, which is consistent with a hole density of $10^{13} \mathrm{~cm}^{-2}$.

\section{Discussion}

The stability of the doping by $\mathrm{HNO}_{3}$ is expressed as a fraction of the recovered sheet resistance at different temperatures. In a simple model, the observed increase in sheet resistance with annealing temperature can be explained by the desorption of molecules from the graphene surface, for which a similar behavior in the normalized data of figures $1 \mathrm{~d}, 1 \mathrm{e}$, and $1 \mathrm{f}$ would be expected. However, our data show significant deviation from this model, suggesting a combination of doping mechanisms is instead involved. From the XPS and Raman spectra, we 
infer that the doping is not exclusively related to absorbed $\mathrm{NO}_{3}{ }^{-}$, as suggested in literature, ${ }^{24}$ but also to a contribution from other adsorbed molecules or atoms, without further significant alteration of the graphene lattice.

We propose the following model based on the evolution of both XPS and Raman spectra, and on the work by Martyna et al. ${ }^{34}$ The $\mathrm{HNO}_{3}$ molecules physisorb onto the graphene sheet, without breaking any $\mathrm{C}-\mathrm{C}$ bonds. The $\mathrm{HNO}_{3}$ molecule then dissociates into three groups, two radicals $\mathrm{NO}_{2}{ }^{0}$ and $\mathrm{NO}_{3}{ }^{0}$ and a water molecule,

$$
2 \mathrm{HNO}_{3}=\mathrm{NO}_{2}{ }^{0}+\mathrm{NO}_{3}{ }^{0}+\mathrm{H}_{2} \mathrm{O}
$$

All three species are physisorbed. The two radicals are calculated to have a singly occupied state below the Fermi energy of the graphene layer. ${ }^{34}$ This allows two electrons to transfer from graphene into these states, creating two holes in the graphene and causing $p$-type doping. The radicals become anions, $\mathrm{NO}_{2}{ }^{-}$and $\mathrm{NO}_{3}{ }^{-}$, and they become bound to the graphene holes by Coulomb attraction. However, they do not form chemisorptive bonds to adjacent carbon atoms, the bond length is too large for this, and the adjacent carbon atoms do not pucker upwards as required to allow the formation of these extra bonds. Thus, the physisorptive sites do not cause an increase in the Raman D peak (as observed).

In addition to $\mathrm{NO}_{3}{ }^{-}$adsorption/desorption, the stability assessment reveals an additional factor when doping graphene with concentrated $\mathrm{HNO}_{3}$ at high temperatures. We ascribe this to oxygenrich groups decorating the graphene layer which are present mostly at higher concentration and temperature of the $\mathrm{HNO}_{3}$ doping process. Concentrated $\mathrm{HNO}_{3}$ is known to be a strong oxidant 
which induces the functionalization of graphitic carbon, especially at high temperatures. This was observed on graphite ${ }^{35}$ and on multilayer graphene, ${ }^{33}$ where oxygen is covalently bonded to the graphene lattice as hydroxyl and carboxyl groups. Given our conditions (monolayer graphene transferred onto a silica substrate), we cannot directly determine by XPS the binding of oxygen species to graphene: The $\mathrm{O} 1 s$ is dominated by the substrate oxygen, while the peaks referring to carbon bound to oxygen in the $\mathrm{C} 1 s$ spectrum are covered by the overlapping signal coming from residual PMMA after transfer.

Interestingly, we do not observe an increase in the D-peak in the Raman spectrum. We cannot completely exclude that the doping is not introducing any defects in the lattice, as doped graphene presents a lower $I_{D} / I_{G}$ in respect to undoped graphene with the same density of defects. ${ }^{36}$ As a control, we immersed a sample of graphene in $65 \%$ at $52{ }^{\circ} \mathrm{C}$ for 2 hours, and the D peak is still not found to increase in intensity (Fig. 3b), and neither after annealing at $130{ }^{\circ} \mathrm{C}$.

Similar to our case, it has been shown that exposure of graphene to oxygen at high temperatures (below $300{ }^{\circ} \mathrm{C}$ ) leads to stable adsorption without a significant increase in the D-peak. ${ }^{37}$ Liu et al. ${ }^{38}$ have shown that annealing monolayer graphene in oxygen to $200-300^{\circ} \mathrm{C}$ for 2 hours, partially etched the layer, but also induced a high hole carrier density $\left(\sim 2 \cdot 10^{13} \mathrm{~cm}^{-2}\right)$. During temperature ramping, the doping manifests first, while the etching (increase of D-peak) occurs at higher temperatures. The doping is therefore not due to damaging of the honeycomb lattice. The stable doping component can be attributed either to 1) oxygen (or an oxygen group) covalently bonded to graphene, consistent with the doping stability but less so with the low D-peak; or to 2) oxygen (or an oxygen group) behaving as a charge-transfer complex, consistent with the low Dpeak, but with the doping stability being less expected. Reports in literature describe cases of oxygen functionalization with minor graphene lattice damage. ${ }^{39,40}$ 
The doping of graphene by $\mathrm{HNO}_{3}$ is therefore unstable when only caused by molecular adsorbates of $\mathrm{NO}_{3}{ }^{-}$but high doping stability is achieved under conditions where the binding or adsorption of oxygen is induced. At acid concentrations below 50\%, the obtained doping is mainly unstable. In contrast, using higher concentrations and temperatures, the doping becomes stable. This explains figure 1d, which show a significant variation of normalized doping stability when graphene is doped with acid concentrations lower or higher than 50\%. By increasing the acid temperature, the doping stability increases, indicating that the doping component related to oxidation is increased. This hypothesis is also confirmed by the change in slope of the $52^{\circ} \mathrm{C}$ acid temperature line in figure $1 \mathrm{f}$ after the annealing at $200{ }^{\circ} \mathrm{C}$, in agreement with previous results. ${ }^{39}$ Figure 5a compares the stability data of $\mathrm{HNO}_{3}$-doped graphene with similar studies done on $\mathrm{I}_{2}$ and $\mathrm{MoO}_{3}$-doped graphene. ${ }^{14}$ The plot relates the absolute values of sheet resistance of doped graphene before and after an annealing at $130{ }^{\circ} \mathrm{C} . \mathrm{MoO}_{3}$ and $\mathrm{I}_{2}$ are two extreme cases of highly stable and highly unstable dopants respectively. Although $\mathrm{MoO}_{3}$ doping gives high stability, ${ }^{14}$ the absolute values of sheet resistance are still relatively high. $\mathrm{HNO}_{3}$, in the case of annealed and concentrated acid, presents an intermediate stability behavior, along with a significant decrease in absolute sheet resistance.

Figure $5 \mathrm{~b}$ compares the transparency between the same doping methods. $\mathrm{HNO}_{3}$-doped graphene enhances the transparency from $97.1 \%$ of pristine graphene to $97.4 \%$ of transmission at $550 \mathrm{~nm}$; $\mathrm{I}_{2}$-doped graphene from $97.1 \%$ to $97.6 \%$ of transmission at $550 \mathrm{~nm}$, while an adlayer of $5 \mathrm{~nm}$ of $\mathrm{MoO}_{3}$ reduces the transparency (from $97.1 \%$ to $95.4 \%$ of transmission at $550 \mathrm{~nm}$ ), especially towards the UV region. The improved transparency of the acid can partly be attributed to the removal of polymer contaminations. ${ }^{24}$ 
It is worth noting that, even though the monolayer-graphene sheet resistance in this work is comparable with the lowest reported to date, ${ }^{8}$ it may be possible to further reduce this value by changing or pretreating the substrate ${ }^{41,42,43}$ or by modifying the CVD graphene quality.

\section{Conclusion}

We have demonstrated that low sheet resistances and stability can be obtained simultaneously in adsorptive-doped graphene. We have analyzed the doping of graphene with $\mathrm{HNO}_{3}$ at various concentrations and temperatures, and its stability upon annealing in vacuum. We have found that graphene doped with concentrated $\mathrm{HNO}_{3}$ at high temperatures exhibits both a sheet resistance as low as $180 \Omega / \mathrm{sq}$ and an improved stability, i.e. less than $2 \%$ recovery of the doping resistance drop when annealing at $130{ }^{\circ} \mathrm{C}$ for $30 \mathrm{~min}$. We find the doping and stability to arise from a combination of doping mechanism. Upon exposure to $\mathrm{HNO}_{3}$ at mild conditions, graphene adsorbs unstable $\mathrm{NO}_{3}{ }^{-}$molecules which easily desorb at low temperatures. Conversely, doping at high concentrations and temperatures creates stable $p$-type doping by the adsorption or functionalization of oxygen. This report of both stable and effective doping reveals a promising route toward ITO replacement by graphene.

\section{AUTHOR INFORMATION}

\section{Corresponding Author}

*Email: LD423@cam.ac.uk

\section{Notes}

The authors declare no competing financial interest. 


\section{ACKNOWLEDGMENT}

We acknowledge the European project GRAFOL for funding. L.D. acknowledges funding from EPSRC. R.S.W. acknowledges a research fellowship from St. John's College, Cambridge and a Marie Skłodowska-Curie Individual Fellowship (Global) under grant ARTIST (no. 656870) from the European Union's Horizon 2020 research and innovation programme. X.W. acknowledges a scholarship from the Cambridge Overseas Trust and the Learning, and Research Funding from St

John's College Cambridge. W.A. acknowledges funding from EPSRC Cambridge NanoDTC (EP/L015978/1). H.S. acknowledges a research fellowship from the Japanese Society for the Promotion of Science (JSPS). C.C. acknowledges MIUR (PRIN 2010-2011 No. 2010N3T9M4), the project ABNANOTECH (supported by MIUR, 'Progetto Premiale 2012'), and In-Kind (PIK) EX- PROREL for financial support. 
Figure 1: $(\mathrm{a}, \mathrm{b}, \mathrm{c})$ sheet resistance of graphene doped with different (a) $\mathrm{HNO}_{3}$ concentrations, (b) dipping times, and (c) acid temperatures. The left axis refers to values normalized to the undoped sheet resistance, which are consistent for a range of different undoped sheet resistances (2600$3500 \Omega /$ sq.). The right axis shows the indicative absolute sheet resistance of an average device. $(d, e, f)$ variation of sheet resistance upon annealing normalized to the variation of sheet resistance upon doping, i.e. the ratio of sheet resistance recovered upon annealing. The data refers to graphene doped with different (d) $\mathrm{HNO}_{3}$ concentrations, (e) dipping times, and (f) acid temperatures and the annealing is performed sequentially at $70{ }^{\circ} \mathrm{C}, 130{ }^{\circ} \mathrm{C}, 200{ }^{\circ} \mathrm{C}$ and $300{ }^{\circ} \mathrm{C}$ for 30 minutes. The errors are the standard deviation on the average of measurements taken on different devices.

Figure 2: Mobility $(\mu)$ and charge carrier density (n) for graphene as-transferred, doped and postannealed $\left(200{ }^{\circ} \mathrm{C}, 30 \mathrm{~min}\right)$, measured with Van der Pauw geometry Hall devices. The graph regards three samples with different doping: $5 \mathrm{~min}$ of dipping into $22 \%$ (blue) and 65\% (green) of $\mathrm{HNO}_{3}$ solution at $\mathrm{RT}$, and $65 \%$ of $\mathrm{HNO}_{3}$ solution at $52{ }^{\circ} \mathrm{C}$ (orange and red).

Figure 3: (a) Raman spectra of graphene before (pre-annealed) and after doping with $22 \% \mathrm{HNO}_{3}$ solution at RT for $5 \mathrm{~min}, 65 \% \mathrm{HNO}_{3}$ solution at RT for $5 \mathrm{~min}$ and $65 \% \mathrm{HNO}_{3}$ solution heated at $52^{\circ} \mathrm{C}$ for $5 \mathrm{~min}$. (b) Raman spectra of graphene after doping with $65 \% \mathrm{HNO}_{3}$ solution heated at $52^{\circ} \mathrm{C}$ for $120 \mathrm{~min}$, and after post-annealing at $130^{\circ} \mathrm{C}$. The region near the D-peak $\left(\sim 1345 \mathrm{~cm}^{-1}\right)$ has been enlarged by a factor 10. All spectra are normalized by the G-peak intensity and are the average of 3 spectra taken at different positions on the sample. The spectrum in red (postannealed) is taken with a 5 times higher laser intensity $(\sim 7.5 \mathrm{~mW})$.

Figure 4: (a) XPS C $1 s$ spectra of graphene pre-annealed at $180{ }^{\circ} \mathrm{C}$ for 1 hour, and after dipping the sample in $65 \% \mathrm{HNO}_{3}$ solution for $5 \mathrm{~min}$ at $52{ }^{\circ} \mathrm{C}$. (b) XPS $\mathrm{N} 1 s$ spectrum of pre-annealed undoped graphene (top spectrum), and XPS N $1 s$ spectrum of graphene doped with $65 \% \mathrm{HNO}_{3}$ solution for $5 \mathrm{~min}$ at $\mathrm{RT}$ and $52{ }^{\circ} \mathrm{C}$, and with $22 \% \mathrm{HNO}_{3}$ for $5 \mathrm{~min}$ at RT, and the same spectra after post-annealing at $200{ }^{\circ} \mathrm{C}$.

Figure 5: (a) Comparison of sheet resistances after doping and after post-doping annealing for graphene doped with $\mathrm{HNO}_{3}\left(5 \min 52^{\circ} \mathrm{C} 65 \%\right)$, and $\mathrm{MoO}_{3}$ and $\mathrm{I}_{2}$ from a previous work. ${ }^{14}$ The annealing was performed at $130{ }^{\circ} \mathrm{C}$ in vacuum $\left(10^{-6} \mathrm{mbar}\right)$ for $30 \mathrm{~min}$ for all dopants. (b) UVVIS transmittance measurements of as-transferred graphene and $\mathrm{HNO}_{3}$-doped graphene, compared to graphene doped with $\mathrm{MoO}_{3}$ and $\mathrm{I}_{2}$ from a previous work. ${ }^{14} \mathrm{MoO}_{3}$ reduces the transparency of graphene; while $\mathrm{HNO}_{3}$ and $\mathrm{I}_{2}$ slightly increase the light transmittance, probably due to the removal of PMMA residue. 
Figure 1.

a

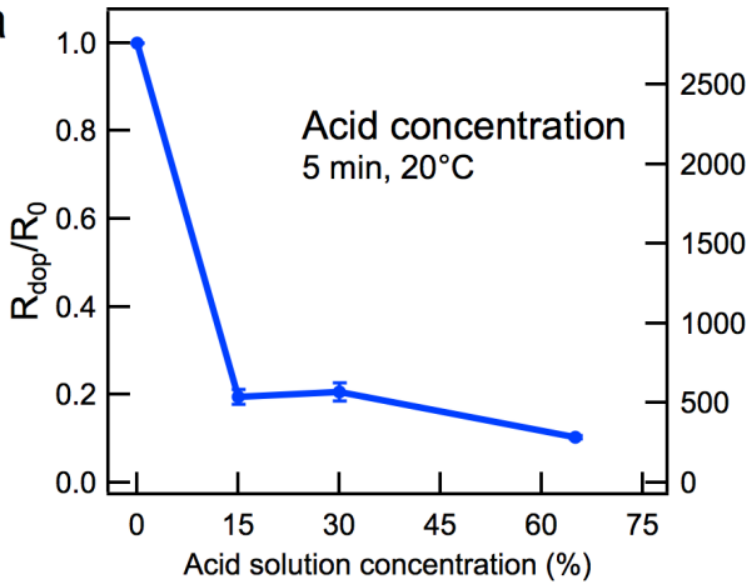

b

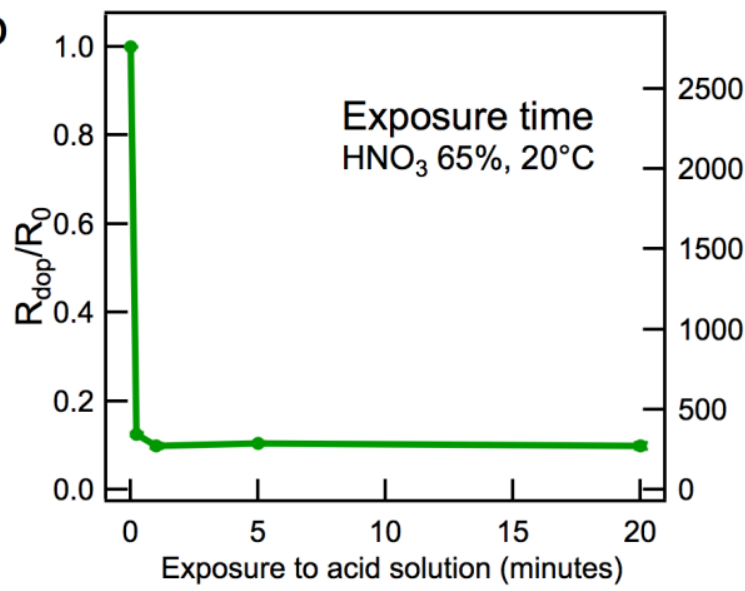

C

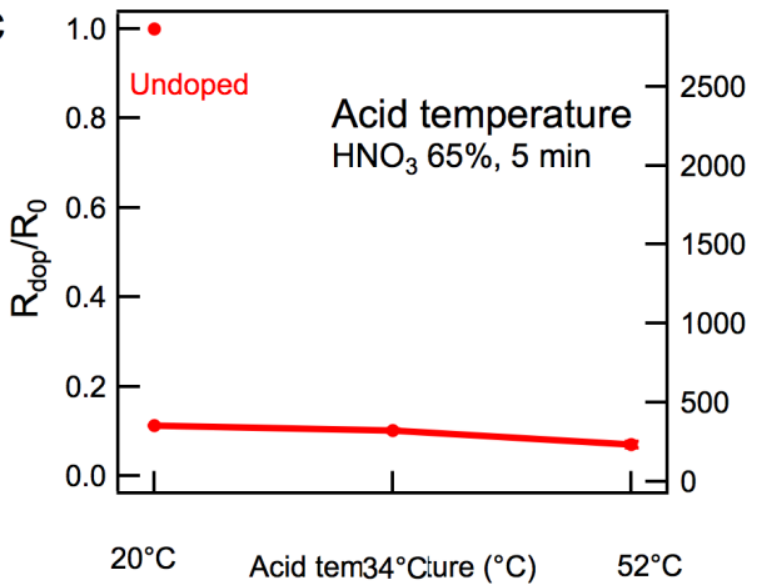

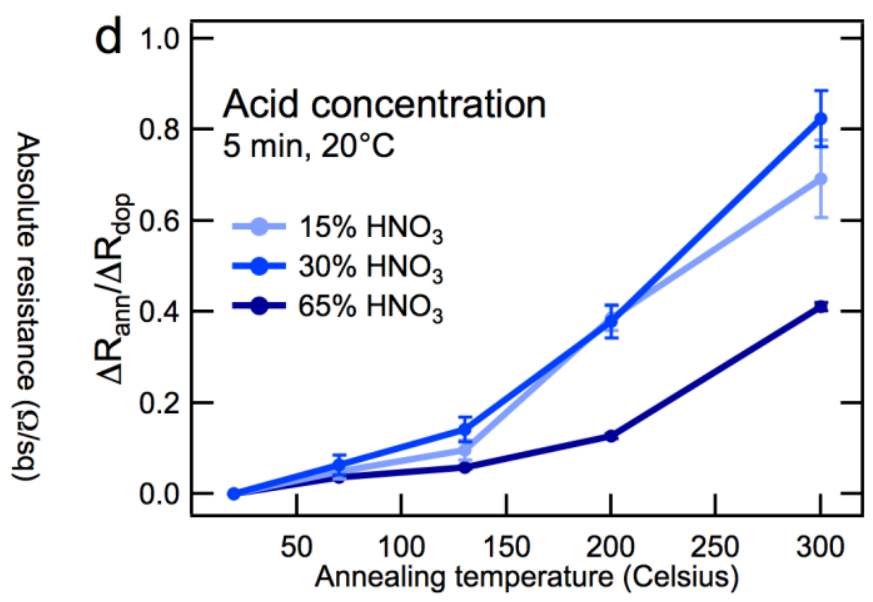
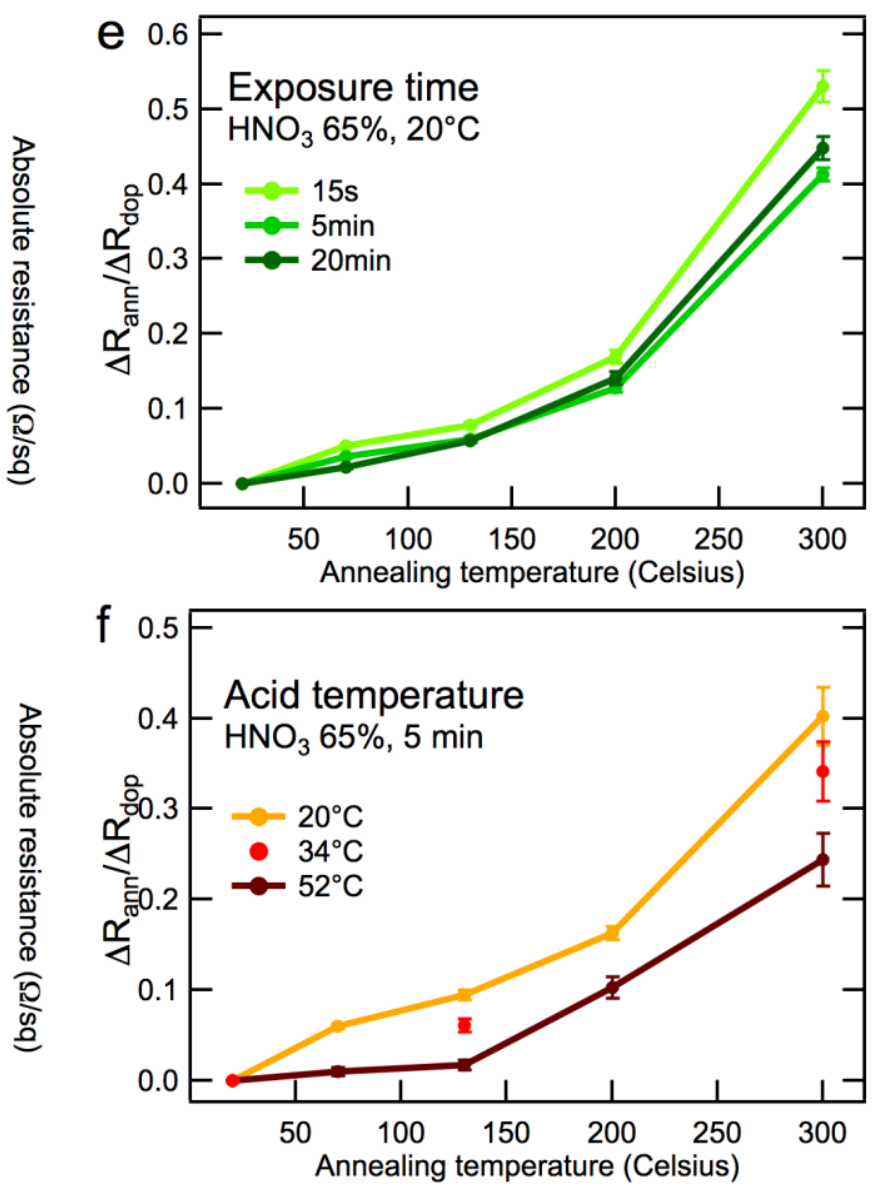
Figure 2.

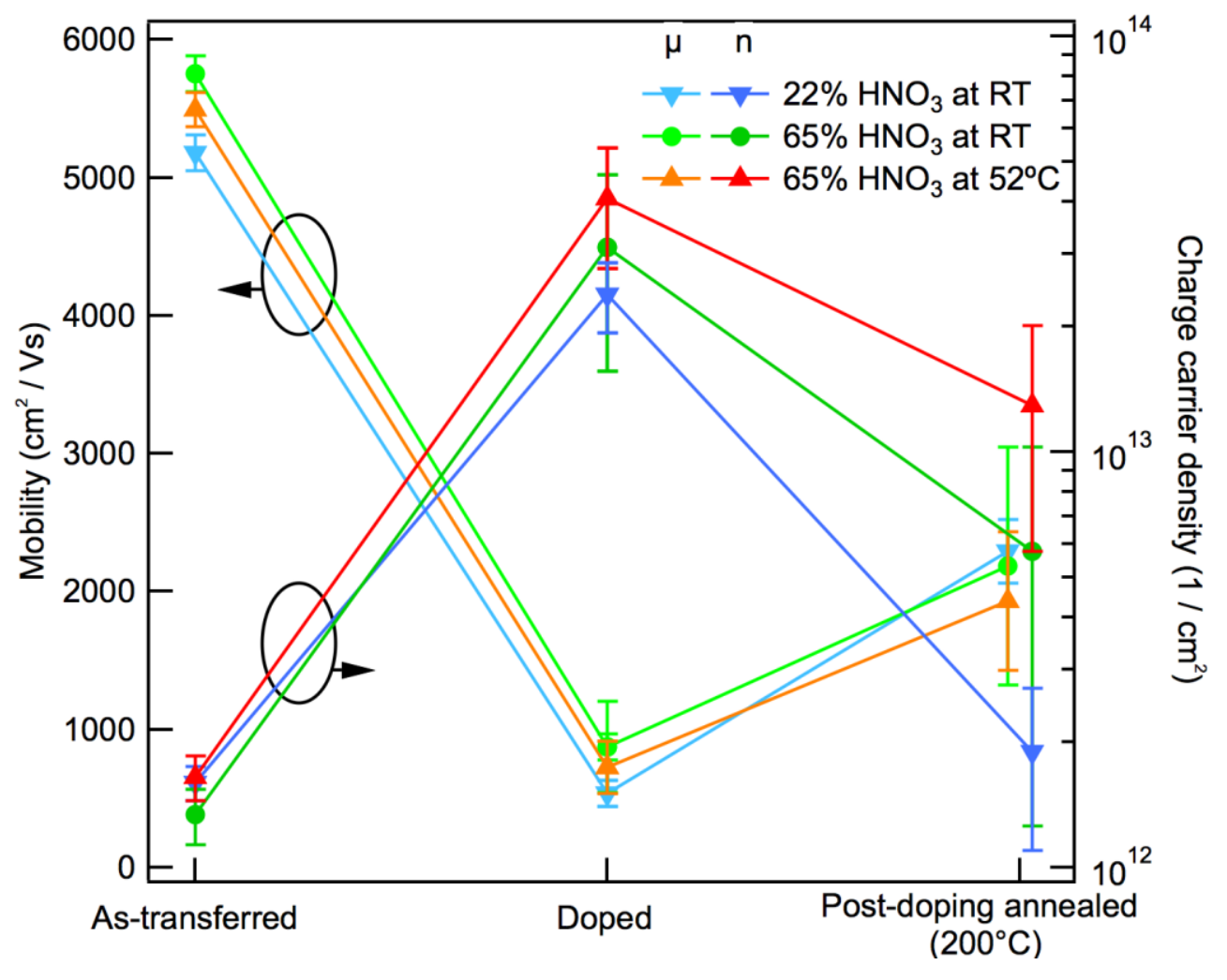


Figure 3.
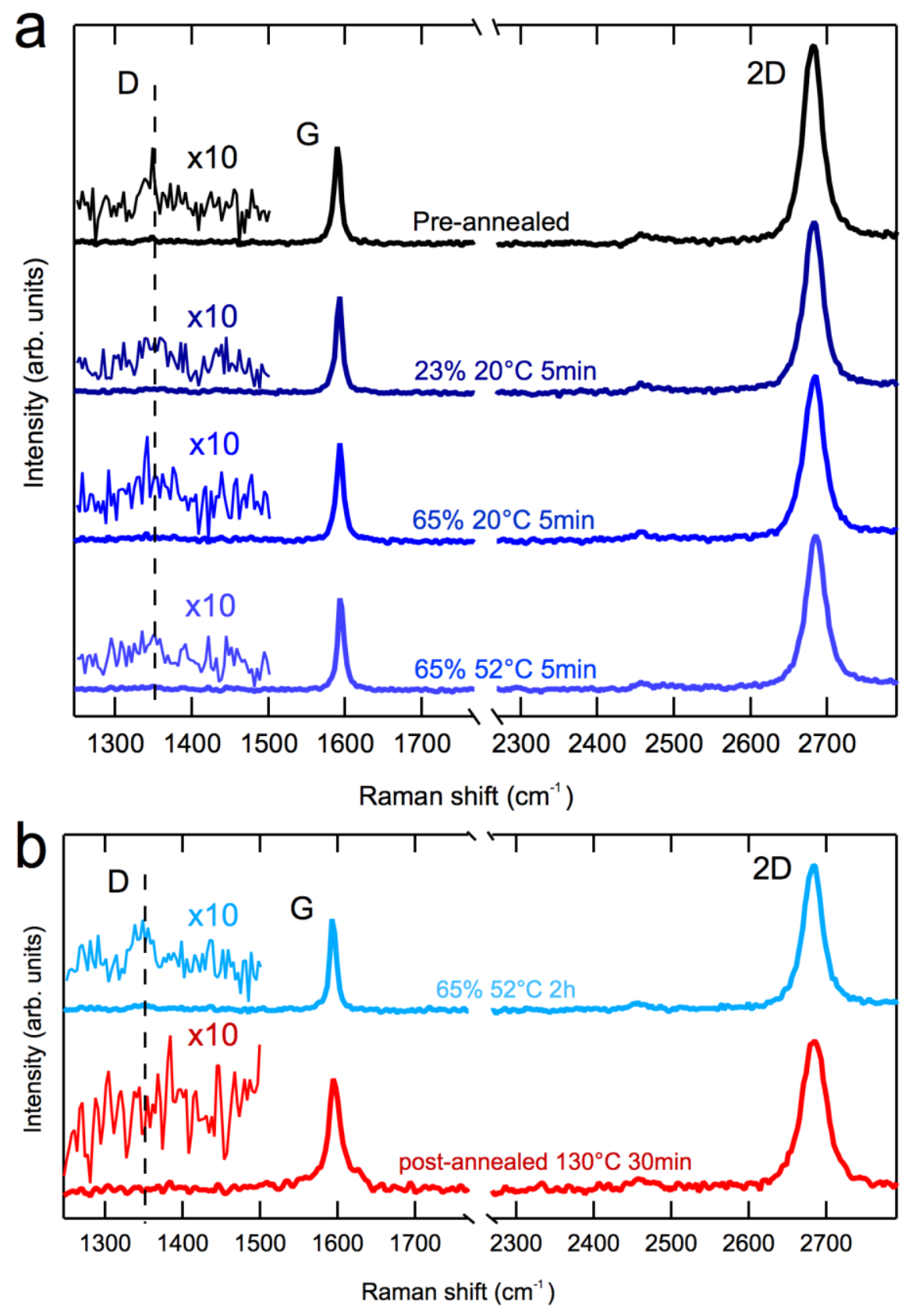
Figure 4.
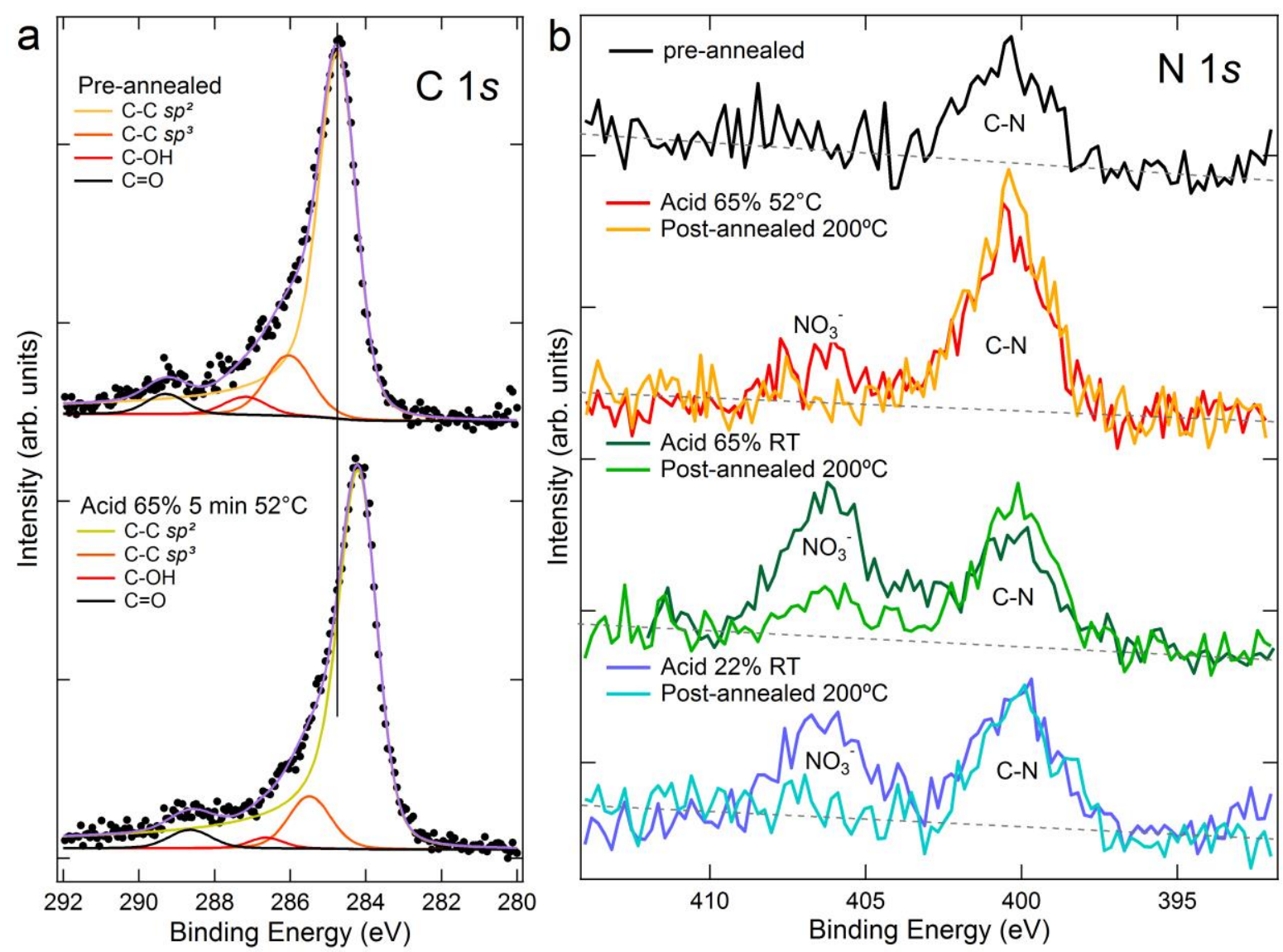
Figure 5.
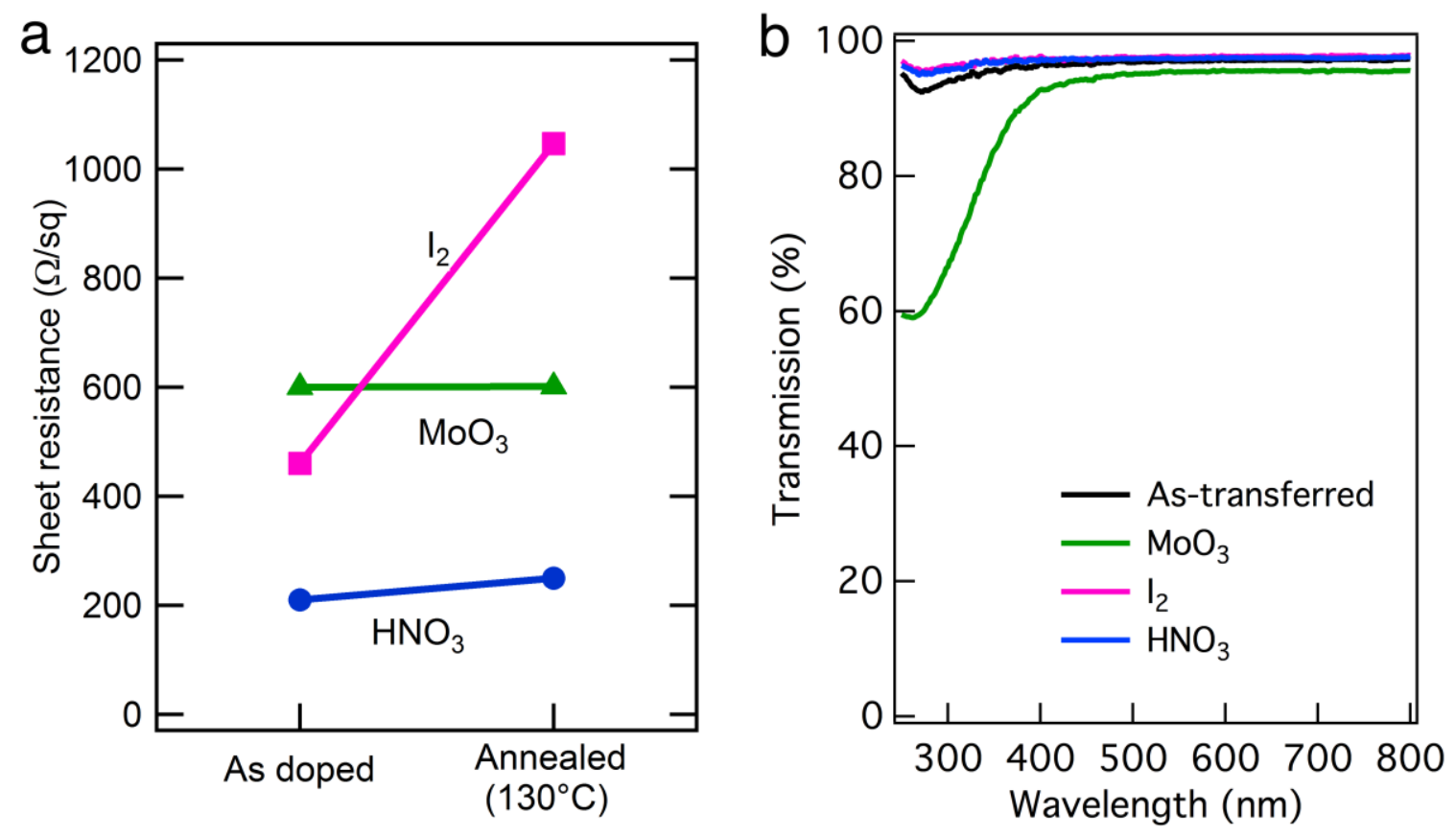
${ }^{1}$ Geim, A.K.; Novoselov, K.S. The rise of graphene. Nature Mater. 2007, 6, 183-91.

${ }^{2}$ Novoselov, K.S.; Fal'ko, V.I.; Colombo, L.; Gellert, P.R.; Schwab, M.G.; Kim, K. A roadmap for graphene. Nature 2012, 490, 192-200.

${ }^{3}$ Hofmann, S.; Braeuninger-Weimer, P.; Weatherup, R. S. CVD-Enabled Graphene Manufacture and Technology. J. Phys. Chem. Lett. 2015, 6, 2714-2721.

${ }^{4}$ El-Kady, M.F.; Kane, R.B. Direct Laser Writing of Graphene Electronics. ACS Nano 2014, 8, 8725-9.

${ }^{5}$ Chien, C.; Hiralal, P.; Wang, D.; Huang, I.; Chen, C.; Chen, C.; Amaratunga, G.A.J. Graphene-Based Integrated Photovoltaic Energy Harvesting/Storage Device. Small 2015, 11, 2929-37

${ }^{6}$ Raccichini, R.; Varzi, A.; Passerini, S.; Scrosati, B.; The role of graphene for electrochemical energy storage. Nature Mater. 2015, 14, 271-9.

${ }^{7}$ Kuruvila, A.; Kidambi, P.; Kling, J.; Wagner, J.B.; Robertson, J.; Hofmann, S.; Meyer, J. Organic light emitting diodes with environmentally and thermally stable doped graphene electrodes. J. Mater. Chem. C 2014, 2, 6940-5.

${ }^{8}$ Bae, S.; Kim, H.; Lee, Y.; Xu, X.; Park, J.; Zheng, Y.; Balakrishnan, J.; Lei, T.; Kim, H.R.; Song, Y.I.; Kim, Y.; Kim, K.S.; Ozyilmaz, B.; Ahn, J.; Hong, B.H.; Iijima, S. Roll-to-roll production of 30-inch graphene films for transparent electrodes. Nat. Nanotech. 2010, 5, 574-8.

${ }^{9}$ Jo, G.; Choe, M.; Lee, S.; Park, W.; Kahng, Y.H.; Lee, T. The application of graphene as electrodes in electrical and optical devices. Nanotechnology 2012, 23, 112001.

${ }^{10}$ Park, H.; Rowehl, J.A.; Kim, K.K.; Bulovic, V.; Kong, J. Doped graphene electrodes for organic solar cells. Nanotechnology 2010, 21, 505204.

${ }^{11}$ Wei, D; Liu, Y; Wang, Y.; Zhang, H.; Huang, L.; Yu, G. Synthesis of N-Doped Graphene by Chemical Vapor Deposition and Its Electrical Properties. Nano Lett. 2009, 9, 1752-8.

${ }^{12}$ Wang, H.; Maiyalagan, T.; Wang, X. Review on Recent Progress in Nitrogen-Doped Graphene: Synthesis, Characterization, and Its Potential Applications. ACS Catatal. 2012, 2, 781-94.

${ }^{13}$ Sinitskii, A.; Dimiev, A.; Corley, D.A.; Fursina, A.A.; Kosynkin, D.V.; Tour, J.M. Kinetics of Diazonium Functionalization of Chemically Converted Graphene Nanoribbons. ACS Nano 2010, 4, 194954.

${ }^{14}$ D’Arsié, L.; Esconjauregui, S.; Weatherup, R.; Guo, Y.; Bhardwaj, S.; Centeno, A.; Zurutuza, A.; Cepek, C; Robertson, J. Stability of graphene doping with $\mathrm{MoO}_{3}$ and $\mathrm{I}_{2}$. Appl. Phys. Lett. 2014, 105, 103103.

${ }^{15}$ Kim, H.; Kim, H.H.; Jang, J.I.; Lee, S.K.; Lee, G.; Han, J.T.; Cho, K. Doping Graphene with an Atomically Thin Two Dimensional Molecular Layer. Adv. Mater. 2014, 26, 8141-6.

${ }^{16}$ Meyer, J.; Khalandovsky, R.; Görrn, P.; Kahn, A. $\mathrm{MoO}_{3}$ Films Spin-Coated from a Nanoparticle Suspension for Efficient Hole-Injection in Organic Electronics. Adv. Mater. 2011, 23, 70-73.

${ }^{17}$ Chen, Z.; Santoso, I.; Wang, R.; Xie, L.F.; Mao, H.Y.; Huang, H.; Wang, Y.Z.; Gao, X.Y.; Chen, Z.K.; Ma, D.; Wee, A.T.S.; Chen, W. Surface transfer hole doping of epitaxial graphene using MoO3 thin film. Appl. Phys. Lett. 2010, 96, 213104.

${ }^{18}$ Esconjauregui, S.; D’Arsié, L.; Guo, Y.; Yang, J.; Sugime, H.; Caneva, S.; Cepek, C.; Robsertson, J. Efficient Transfer Doping of Carbon Nanotube Forests by $\mathrm{MoO}_{3}$. ACS Nano 2015, 9, 10422-10430.

${ }^{19}$ Rinzler, A.G.; Liu, J.; Dai, H.; Nikolaev, P.; Huffman, C.B.; Rodriguez-Macias, F.J.; Boul, P.J.; Lu, A.H.; Heymann, D.; Colber, D.T.; et al. Large-scale purification of single-wall carbon nanotubes: process, product, and characterization. Appl. Phys. A 1998, 67, 29-37.

${ }^{20}$ Chung, D.D.L. Review Exfoliation of graphite. J. Mater. Sci. 1987, 22, 4190-8.

${ }^{21}$ Yu, H.; Jin, Y.; Peng, F.; Wang, H.; Yang, J. Kinetically Controlled Side-Wall Functionalization of Carbon Nanotubes by Nitric Acid Oxidation. Phys. Chem. C 2008, 112, 6758-63.

${ }^{22}$ Wu, X.; Zhong, G.; D'Arsié, L.; Sugime, H.; Esconjauregui, S.; Robertson, A.W.; Robertson J. Growth of Continuous Monolayer Graphene with Millimeter-sized Domains Using Industrially Safe Conditions. Sci. Rep. 2016, 6, 21152. 
${ }^{23}$ Kratzer, M.; Bayer, B.C.; Kidambi, P.R.; Matković, A.; Gajić, R.; Cabrero-Vilatela, A.; Weatherup, R.S.; Hofmann S.; Teichert, C. Effects of polymethylmethacrylate-transfer residues on the growth of organic semiconductor molecules on chemical vapor deposited graphene. Appl. Phys. Lett. 2015, 106, 103101.

${ }^{24}$ Kasry, A.; Kuroda, M.A.; Martyna, G.J.; Tulevski, G.S.; Bol, A.A. Chemical Doping of Large-Area Stacked Graphene Films for Use as Transparent, Conducting Electrodes. ACS Nano 2010, 7, 3839-44.

${ }^{25}$ Xue, J.; Sanchez-Yamagishi, J.; Bulmash, D.; Jacquod, P.; Deshpande, A.; Watanabe, K.; Taniguchi, T.; Jarillo-Herrero, P.; LeRoy B.J. Scanning tunnelling microscopy and spectroscopy of ultra-flat graphene on hexagonal boron nitride. Nat. Mater. 2011, 10, 282-285.

${ }^{26}$ Wang, Y.Y.; Ni, Z.H.; Yu, T.; Shen, Z.X.; Wang, H.M.; Wu, Y.H.; Chen, W.; Wee, A.T.S. Raman Studies of Monolayer Graphene: The Substrate Effect. J. Phys. Chem. C 2008, 112, 10637-40.

${ }^{27}$ Weatherup, R.S.; Dlubak, B.; Hofmann, S. Kinetic Control of Catalytic CVD for High-Quality Graphene at Low Temperatures. ACS Nano 2012, 6, 9996-10003.

${ }^{28}$ Das, A.; Pisana, S.; Chakraborty, B.; Piscanec, S.; Saha, S.K.; Waghmare, U.V.; Novoselov, K.S.; Krishnamurthy, H.R.; Geim, A.K.; Ferrari, A.C.; Sood, A.K. Monitoring dopants by Raman scattering in an electrochemically top-gated graphene transistor. Nat. Nanotech. 2008, 3, 210-5.

${ }^{29}$ Lucchese, M.M.; Stavale, F.; Martins Ferreira, E.H.; Vilani, C.; Moutinho, M.V.O.; Capaz, R.B.; Achete, C.A.; Jorio, A. Quantifying ion-induced defects and Raman relaxation length in graphene. Carbon 2010, 48, 1592-7.

${ }^{30}$ Lin, Y.; Lu, C.; Yeh, C.; Jin, C.; Suenaga, K.; Chiu, P. Graphene Annealing: How Clean Can It Be? Nano Lett. 2012, 12, 414-9.

${ }^{31}$ Bouleghlimat, E.; Davies, P.R.; Davies, R.J.; Howarth, R.; Kulhavy, J.; Morgan D.J. The effect of acid treatment on the surface chemistry and topography of graphite. Carbon 2013, 61, 124-33.

${ }^{32}$ Shin, D.; Lee, J.H.; Kim, Y.; Yu, S.M.; Park, S.; Yoo, J. A role of $\mathrm{HNO}_{3}$ on transparent conducting film with single-walled carbon nanotubes. Nanotechnology 2009, 20, 475703.

${ }^{33}$ Das, S.; Sudhagar, P.; Ito, E.; Lee, D.; Nagarajan, S.; Lee, S.Y.; Kang, Y.S.; Choi, W. Effect of HNO3 functionalization on large scale graphene for enhanced tri-iodide reduction in dye-sensitized solar cells. $J$. Mater. Chem. 2012, 22, 20490-7.

${ }^{34}$ Nistor, R.A.; Newns, D.M.; Martyna, G.J. The Role of Chemistry in Graphene Doping for CarbonBased Electronics. ACS Nano 2011, 5, 3096-103.

${ }^{35}$ Burgess, R.; Buono, C.; Davies, P.R.; Davies, R.J.; Legge, T.; Lai, A.; Lewis, R.; Morgana, D.J.; Robinson, N.; Willock, D.J. The functionalisation of graphite surfaces with nitric acid: Identification of functional groups and their effects on gold deposition. J. Catal. 2015, 323, 10-18.

${ }^{36}$ Bruna, M.; Ott, A.K.; Ijäs, M.; Yoon, D.; Sassi, U.; Ferrari, A.C. Doping Dependence of the Raman Spectrum of Defected Graphene. ACS Nano 2014, 8, 7432-7441.

${ }^{37}$ Piazza, A; Giannazzo, F.; Buscarino, G.; Fisichella, G.; La Magna, A.; Roccaforte, F.; Cannas, M.; Gelardi, F.M.; Agnello, S. Graphene p-Type Doping and Stability by Thermal Treatments in Molecular Oxygen Controlled Atmosphere, J. Phys. Chem. C 2015, 119, 22718.

${ }^{38}$ Liu, L.; Ryu, S.; Tomasik, M.R.; Stolyarova, E.; Jung, N.; Hybertsen, M.S.; Steigerwald, M.L.; Brus, L.E.; Flynn, G.W. Graphene Oxidation: Thickness-Dependent Etching and Strong Chemical Doping. Nano Lett. 2008, 8, 1965

${ }^{39}$ Hossain, M.Z.; Johns, J.E.; Bevan, K.H.; Karmel, H.J.; Liang, Y.T.; Yoshimoto, S.; Mukai, K.; Koitaya, T.; Yoshinobu, J.; Kawai, M.; Lear, A.M.; Kesmodel, L.L.; Tait, S.L.; Hersam, M.C. Chemically homogeneous and thermally reversible oxidation of epitaxial graphene, Nat. Chem. 2012, 4, 305.

${ }^{40}$ Li, J.L.; Kudin, K.N.; McAllister, M.J.; PrudÕhomme, R.K.; Aksay, I.A.; Car, R. Oxygen-Driven Unzipping of Graphitic Materials, Phys. Rev. Lett. 2006, 96, 176101.

${ }^{41}$ Wang, R.; Wang, S.; Zhang, D.; Li, Z.; Fang, Y.; Qiu, X. Control of carrier type and density in exfoliated graphene by interface engineering. ACS Nano 2011, 1, 408-412. 
${ }^{42}$ Wang, Q.H.; Jin, Z.; Kim, K.K.; Hilmer, A.J.; Paulus, G.L.C.; Shih, C.; Ham, M.H.; SanchezYamagishi, J.D.; Watanabe, K.; Taniguchi, T.; Kong, J.; Jarillo-Herrero P.; Strano, M.S. Understanding and controlling the substrate effect on graphene electron-transfer chemistry via reactivity imprint lithography. Nat. Chem. 2012, 4, 724-732.

${ }^{43}$ Weatherup, R.S.; D'Arsié, L.; Cabrero-Vilatela, A.; Caneva, S.; Blume, R.; Robertson, J.; Schlögl, R.; Hofmann S. Long-Term Passivation of Strongly Interacting Metals with Single-Layer Graphene. J. Am. Chem. Soc. 2015, 137, 14358-14366. 